ACCEPTED MANUSCRIPT

\title{
Rotating spoke instabilities in a wall-less Hall thruster: Experiments
}

To cite this article before publication: Stephane Mazouffre et al 2019 Plasma Sources Sci. Technol. in press https://doi.org/10.1088/1361$\underline{6595 / \mathrm{ab} 07 \mathrm{fc}}$

\section{Manuscript version: Accepted Manuscript}

Accepted Manuscript is "the version of the article accepted for publication including all changes made as a result of the peer review process, and which may also include the addition to the article by IOP Publishing of a header, an article ID, a cover sheet and/or an 'Accepted Manuscript' watermark, but excluding any other editing, typesetting or other changes made by IOP Publishing and/or its licensors"

This Accepted Manuscript is @ 2018 IOP Publishing Ltd.

During the embargo period (the 12 month period from the publication of the Version of Record of this article), the Accepted Manuscript is fully protected by copyright and cannot be reused or reposted elsewhere.

As the Version of Record of this article is going to be / has been published on a subscription basis, this Accepted Manuscript is available for reuse under a CC BY-NC-ND 3.0 licence after the 12 month embargo period.

After the embargo period, everyone is permitted to use copy and redistribute this article for non-commercial purposes only, provided that they adhere to all the terms of the licence https://creativecommons.org/licences/by-nc-nd/3.0

Although reasonable endeavours have been taken to obtain all necessary permissions from third parties to include their copyrighted content within this article, their full citation and copyright line may not be present in this Accepted Manuscript version. Before using any content from this article, please refer to the Version of Record on IOPscience once published for full citation and copyright details, as permissions will likely be required. All third party content is fully copyright protected, unless specifically stated otherwise in the figure caption in the Version of Record.

View the article online for updates and enhancements. 


\title{
Rotating spoke instabilities in a wall-less Hall thruster: Experiments.
}

\section{S. Mazouffre, L. Grimaud, S. Tsikata} ICARE, CNRS, 1C avenue de la Recherche Scientifique, 45071 Orléans, France.

E-mail: stephane.mazouffre@cnrs-oleans.fr

\section{K. Matyash, R. Schneider}

Ernst-Moritz-Arndt University, Greifswald, D-17487, Germany.

\begin{abstract}
Properties of rotating spokes have been investigated in the plasma discharge of the $200 \mathrm{~W}$-class permanent magnet ISCT200 Hall thruster in wall-less configuration under various operating conditions. For comparison purposes, the Hall thruster was also operated in the standard configuration. High-speed camera imaging combined with sophisticated image processing allowed the observation of the plasma inhomogeneity dynamics and the determination of characteristics such as mode number, sizes, rotation frequency and propagation velocity. The time varying $\mathrm{Xe}^{+}$ion azimuthal velocity distribution function has been measured in front of the anode of the wall-less thruster by means of laser-induced fluorescence spectroscopy in photoncounting regime. Experiments show ions move at the thermal speed on average, yet some ions have a negative velocity, i.e. they rotate in the direction opposite to the $\mathrm{E} \times \mathrm{B}$ drift, in agreement with numerical simulations performed with a 3D-3V PIC code.
\end{abstract}

Submitted to: Plasma Sources Sci. Technol. 
Rotating spoke instabilities in a wall-less Hall thruster: Experiments.

\section{Introduction}

Rotating plasma inhomogeneities, often termed "rotating spokes", are observed in various types of magnetized low pressure plasma discharges created in a crossed electric and magnetic field configuration [1-4]. Over the past ten years, such structures have been experimentally investigated e.g. in plasma devices like magnetrons [5-10], Hall thrusters [11-18] and plasma columns $[19,20]$. There is a great deal of interest in studying rotating plasma structures for mainly two reasons. Firstly, such instabilities appear to be a very general phenomenon in low-pressure, low-temperature, partially-magnetized plasma discharges, in which their origin and governing mechanisms are not yet fully understood. Secondly, these large-scale, low-frequency rotating instabilities appear to play a role in the transport of charged particles across the magnetic field lines. As a consequence, rotating spokes may influence characteristics and performance of plasma devices and plasma technologies used for applications such as thin-film deposition and spacecraft propulsion.

In this work, we experimentally examine the properties of low-frequency rotating plasma instabilities in the discharge of low power Hall thrusters (HTs). Hall thrusters are electric propulsion devices for satellites and spacecraft that rely on a low-pressure plasma discharge in cross-field configuration to ionize and accelerate a propellant gas [21-23]. A HT is comprised of an anode placed at the back of a coaxial annular dielectric channel and an external thermionic cathode. The propellant gas, typically xenon, is introduced at the back of the channel. Magnetizing coils or permanent magnets, incorporated into a magnetic circuit, generate a radially-directed magnetic field, the strength of which is maximum at the channel exhaust. The magnetic field magnitude is set to confine electrons without affecting ion trajectories. The electric potential drop occurs in the final section of the channel owing to the low axial mobility of electrons in this restricted area. The electric field, which extends between the interior and the exterior of the channel, governs the propellant atoms ionization and the ion acceleration, namely, the thrust and the specific impulse. The combination of the radial magnetic field with the axial electric field generates an $E \times B$ electron drift in the azimuthal direction, the so-called Hall current. This drift is responsible for the very efficient ionization of neutral atoms inside the channel. Thruster operation is also dependent on the large fraction of electrons emitted by the cathode which flow downstream to neutralize the ion beam. HTs produce a large thrust-to-power ratio $(\sim 60 \mathrm{mN} / \mathrm{kW})$ and a large thrust density $(\geq 30$ $\left.\mathrm{N} / \mathrm{m}^{2}\right)$ with a specific impulse $(\leq 2000 \mathrm{~s})$ high enough to guarantee substantial propellant mass savings. The thrust efficiency is about $50 \%$ for low and moderate power HTs $(\leq 1 \mathrm{~kW})$, but it is in excess of $60 \%$ for high-power devices. Hall thrusters are therefore well-suited for various types of maneuvers like orbit transfer, drag compensation, orbit maintenance and attitude control.

Two different configurations of the permanent magnet $200 \mathrm{~W}$-class ISCT200 thruster have been employed for this study: standard (ST) and wall-less (WL). In the ST configuration, the plasma discharge is mostly confined inside the ceramic channel, whereas in the WL mode, ionization and acceleration take place outside the channel. A WL configuration makes easier the access to the plasma discharge and it allows examination of the plasma properties without the influence of wall processes. There are a number of key objectives of this work, namely: to characterize the dynamics of rotating plasma structures in the $E \times B$ discharge of a lowpower HT and to study the impact of operating conditions on properties of the structures, to compare the features of rotating structures in conventional and wall-less Hall thrusters, to compare results with observations in high-power devices and finally, to compare experiments with computer simulation outcomes. Two diagnostic techniques have been employed to examine the rotating spoke properties in this work. High-speed camera imaging has been used to capture the rotating spoke motion and transformation for various thruster operating conditions. Image processing with sophisticated algorithms allowed the determination of the rotation velocity, direction and frequency, the plasma structure shape and sizes and the mode number. Time-resolved laserinduced fluorescence spectroscopy in the near infra-red has been employed to record the temporal evolution of the ion azimuthal velocity component during a spoke rotation. 


\section{Experimental apparatus}

\section{1. $200 \mathrm{~W}$ Hall thrusters}

Experiments have been performed with the low-power ISCT200 Hall thruster, a $200 \mathrm{~W}$-class Hall thruster which uses permanent magnets for generating the magnetic field instead of helical magnetizing coils [24-26]. Standard (ST) and wall-less (WL) configurations have been employed here. The two versions share many common features in terms of design and architecture. The channel geometry is in the so-called $2 S_{0}$ configuration, in which the channel width-to-mean diameter ratio $h / d$ is twice the standard ratio, where this standard ratio is defined as that of the well-known Russian SPT100 thruster. The inner and outer channel walls are made of Boron nitride-Silica composite $\left(\mathrm{BN}-\mathrm{SiO}_{2}\right)$. Small SmCo permanent magnets combined with a soft iron magnetic circuit generate the magnetic field. The field topology is the same for the two configurations, with magnetic field lines perpendicular to the channel walls at the channel outlet. The magnetic field is directed inwards, and consequently the $E \times B$ electron drift propagates clockwise. The magnetic field strength is highest on the channel axis at the channel exit plane with $B_{\max } \approx 300 \mathrm{G}$. A porous mullite ceramic placed at the back of the channel serves as the gas injector.

A heated hollow cathode with a disc-shaped $\mathrm{LaB}_{6}$ emitter was used with the two thrusters [27]. The cathode, which produces the electron current for discharge balance and ion beam neutralization, is located in the plume far-field $50 \mathrm{~cm}$ away from the channel exit plane nearby the thruster axis to avoid breaking the cylindrical symmetry [28]. The cathode is electrically connected to the thruster anode and floating, like the thruster body. Xenon (Xe) was used as the propellant gas for both the thruster discharge and the cathode discharge. The cathode has been operated with a constant Xe mass flow rate of $0.2 \mathrm{mg} / \mathrm{s}$. Typically, about $150 \mathrm{~W}$ electrical power is applied to the cathode to sufficiently heat the emitting element.

The main differences between the two ISCT200 thruster configurations are, in fact, the anode geometry and position. In the standard version, the anode is a stainless steel ring $5 \mathrm{~mm}$ in height and $1 \mathrm{~mm}$ thick. It is placed at the back of the channel against the internal surface of the outer dielectric wall, i.e. far from the magnetic barrier. In contrast, in the wall-less configuration the anode is located in the magnetic barrier, as shown in Fig. 1.

A well-established approach to reduce ion bombardment in the final section of a HT channel, hence prolonging the thruster lifetime, is magnetic shielding, which, in short, consists of preventing the magnetic field lines from crossing the walls in the acceleration region [29-35]. The WL approach is a more drastic alternative to reducing the channel erosion. The principle is to entirely shift the ionization and acceleration regions outside the cavity by moving the anode towards the channel exit plane [36-39]. Figure 1 illustrates the anode architecture and the magnetic field topology in the ST and WL configurations. In the WL mode, the anode is placed at the channel exhaust. To guarantee efficient confinement of electrons downstream of the channel exit plane, thereby maintaining performance at a high level, the magnetic field lines must not intersect the anode [37]. A possible solution is to use a gridded anode with a curved surface in such a way that the anode 
Rotating spoke instabilities in a wall-less Hall thruster: Experiments.

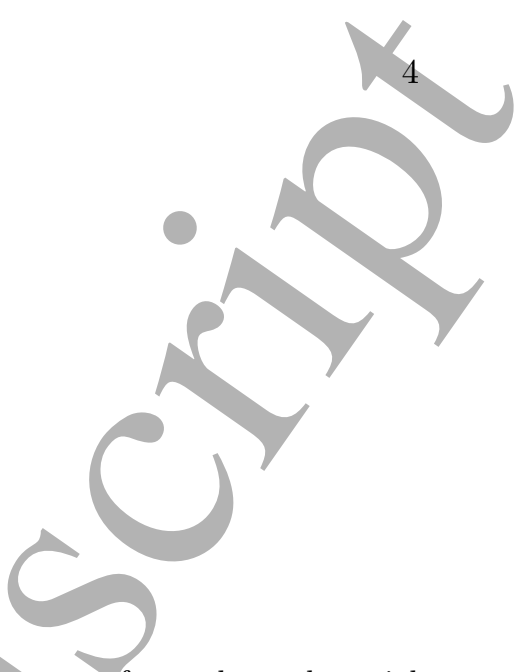

Figure 2. Front view diagram of a HT with the reference system for angles and spatial coordinates. Also shown are the $\mathbf{E}$ and $\mathbf{B}$ vectors and the drift velocity along with the LIF geometry.

surface is parallel to the magnetic field lines [40]. Here the anode is a $1 \mathrm{~mm}$-thick grid with $3 \mathrm{~mm}$ diameter circular holes. The anode transparency is 0.68 , which allows a homogeneous propellant gas distribution outside the channel. From a more fundamental perspective, a wall-less ion source provides an ideal platform for the study of cross-field discharge configurations with electrostatic probes and with laser-aided diagnostics. The access it provides to key regions of the plasma facilitates a thorough investigation of plasma instabilities and small scale turbulence for a better understanding of the discharge physics and anomalous electron transport. Additionally, it allows the study of these phenomena without the influence of wall processes such as secondary electron emission and sputtering as the dielectric channel does not play any role. A WL Hall thruster is also an ideal configuration to make comparisons between experiments and computer simulations. Due to its simplified architecture, boundary conditions are easier to model. For instance the radial boundary conditions correspond to the grounded vacuum chamber walls. A WL source therefore permits more accurate and more reliable numerical outcomes to be obtained.

The radial boundary conditions are in fact well-defined as they correspond to the wall of the vacuum chamber. The potential is zero (walls are grounded) and charged particles recombine when reaching the walls. In the model, all particles are absorbed. The current is not fixed, but it is self-consistently calculated. The simulated chamber is of course much smaller than the real one. However, as long as the boundary is placed far enough from the thruster we can assume that it does not strongly influenees the numerical results. The main advantage of the WL case compared to the standard one is the fact that the channel, which is dielectric, does not have to be accounted for. In other words, we do not have to account for complicated plasma-wall interactions like secondary electron emission or partial recombination.

\subsection{Vacuum chamber and coordinate system}

All experiments have been performed in the cryogenically-pumped NExET (New Experiments on Electric Thrusters) vacuum chamber, a stainless steel tank $1.8 \mathrm{~m}$ in length and $0.8 \mathrm{~m}$ in diameter. Primary pumping is provided by a dry pump that evacuates $400 \mathrm{~m}^{3} / \mathrm{h}$. A $350 \mathrm{l} / \mathrm{s}$ turbomolecular pump further pumps the chamber down to $10^{-6}$ mbar- $\mathrm{N}_{2}$, by absorbing water vapor and light gases such as $\mathrm{N}_{2}, \mathrm{O}_{2}$ and $\mathrm{H}_{2}$. The cryogenic pump absorbs gases such as xenon and krypton. The pumping speed is around $8,000 \mathrm{l} / \mathrm{s}$ when the $0.5 \mathrm{~m}^{2}$ cold panel is sustained at $35 \mathrm{~K}$. The overall pump stack guarantees a background pressure as low as $2 \times 10^{-5}$ mbar-Xe during operation of a $400 \mathrm{~W}$ input power plasma source. A large water-cooled screen covered with graphite tiles is mounted at the rear of the chamber to absorb part of the ion beam energy, thereby reducing the thermal load on the cryogenic surface. The chamber is equipped with several observation windows, access ports and vacuum feed-through connectors for power lines, gas lines and diagnostic tools. A large front door enables easy access to the interior of the vessel. 
Rotating spoke instabilities in a wall-less Hall thruster: Experiments.

Figure 2 shows the reference system for angles and Cartesian coordinates used in this study. Angles are defined according to the unit circle. Also shown in Fig. 2 are the electric field and magnetic field vectors as well as the associated $E \times B$ electron drift velocity. Electrons rotate clockwise in the figure, which corresponds to the ISCT200 Hall thruster configuration used in this work.

\section{Diagnostic techniques}

\subsection{LIF spectroscopy}

The time-averaged and time-varying distribution functions of the velocity of metastable $\mathrm{Xe}^{+}$ions have been measured by means of near-infrared Laser-Induced Fluorescence (LIF) spectroscopy. The LIF technique has been extensively described elsewhere, see e.g. [41, 42] and references therein. Only a brief description of the LIF optical train is given here. An amplified tunable single-mode external cavity laser diode is used to excite metatstable $\mathrm{Xe}^{+}$ion at $834.72 \mathrm{~nm}$. The laser wavelength is accurately measured by means of a calibrated wavemeter. A scanning confocal Fabry-Pérot interferometer is used to perform a real-time check of the quality of the laser mode and to detect mode hops. The power of the beam is also continuously monitored. The primary laser beam is modulated by a mechanical chopper at a frequency of around $2 \mathrm{kHz}$ to allow phase sensitive detection of the fluorescence signal. The laser beam is coupled into a single-mode optical fiber and carried into the NExET vacuum chamber. At the exit of the optical fiber, collimation optics form a $1 \mathrm{~mm}$-diameter beam, the power of which is maintained below $5 \mathrm{~mW} / \mathrm{mm}^{2}$ to avoid strong saturation of the optical transition. The fluorescence radiation at $541.91 \mathrm{~nm}$ is collected using a $40 \mathrm{~mm}$-focal length lens that focuses the light onto a $200 \mu \mathrm{m}$ core diameter multimode fiber. The magnification ratio is 1:1. The fluorescence light transported by the fiber is focused onto the entrance slit of a $20 \mathrm{~cm}$-focal length monochromator that isolates the fluorescence line from the rest of the spectrum. A photomultiplier tube serves as a light detector. In time-averaged mode, a lock-in amplifier operating at the chopper frequency is used to discriminate between the fluorescence light and the intrinsic plasma emission. In time-resolved mode, the photon counting technique with a low dark-noise PMT is used to acquire the fluorescence time-series. The geometry of the LIF setup is shown in Fig. 2. The laser beam propagates horizontally in front of the channel exit plane. The laser wave vector $\mathbf{k}$ is collinear to the ion azimuthal velocity on the channel centerline at $\theta=-90^{\circ}$. The fluorescence light is detected at $40^{\circ}$.

\subsection{High-speed camera}

Two versions of the Phantom high-speed digital camera from Vision Research have been employed for real-time imaging of the thruster discharge: the v2512 and the v1612. A speed up to $1 \mathrm{M}$ framesper-second (fps) can be achieved with the two cameras at reduced resolution. The maximum image resolution is $1280 \times 800$ pixels. The minimum exposure time is $1 \mu \mathrm{s}$. Images are saved in grayscale with a 12-bit depth per pixel that yields high dynamic range and excellent image quality.

For high-speed camera imaging of the two configurations of the ISCT200 Hall thruster, the camera has been aligned on the thruster axis and positioned $2 \mathrm{~m}$ away from the channel exit plane. Imaging of the thruster front panel is performed through a $5 \mathrm{~mm}$-thick quartz window.

\section{Image processing}

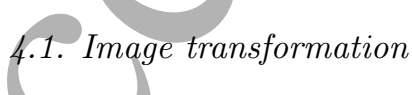

Before processing images, it is necessary to adapt the movie and image format for computer analysis. Several high-speed camera video sizes have been tested. A good compromise between the file size, which defines the computation time, and the number of recorded images that allows for a good statistics during data treatment was found to be in the range [2000-10000] images. Most videos treated in this work comprise 2000 frames and the corresponding file size is around 300 KiloOctets according to camera resolution. The Phantom camera saves raw video in the cine 
Rotating spoke instabilities in a wall-less Hall thruster: Experiments.

Figure 3. Snapshot of the
rotating plasma structure in
the discharge of the ISCT200-
WL thruster operating at $110 \mathrm{~V}$.
Filtered image with a 8 -bit
grayscale.

Figure 3. Snapshot of the
rotating plasma structure in
the discharge of the ISCT200-
WL thruster operating at $110 \mathrm{~V}$.
Filtered image with a 8 -bit
grayscale.

Figure 3. Snapshot of the
rotating plasma structure in
the discharge of the ISCT200-
WL thruster operating at $110 \mathrm{~V}$.
Filtered image with a 8 -bit
grayscale.

Figure 3. Snapshot of the
rotating plasma structure in
the discharge of the ISCT200-
WL thruster operating at $110 \mathrm{~V}$.
Filtered image with a 8 -bit
grayscale.

Figure 3. Snapshot of the
rotating plasma structure in
the discharge of the ISCT200-
WL thruster operating at $110 \mathrm{~V}$.
Filtered image with a 8 -bit
grayscale.

Figure 3. Snapshot of the
rotating plasma structure in
the discharge of the ISCT200-
WL thruster operating at $110 \mathrm{~V}$.
Filtered image with a 8 -bit
grayscale.

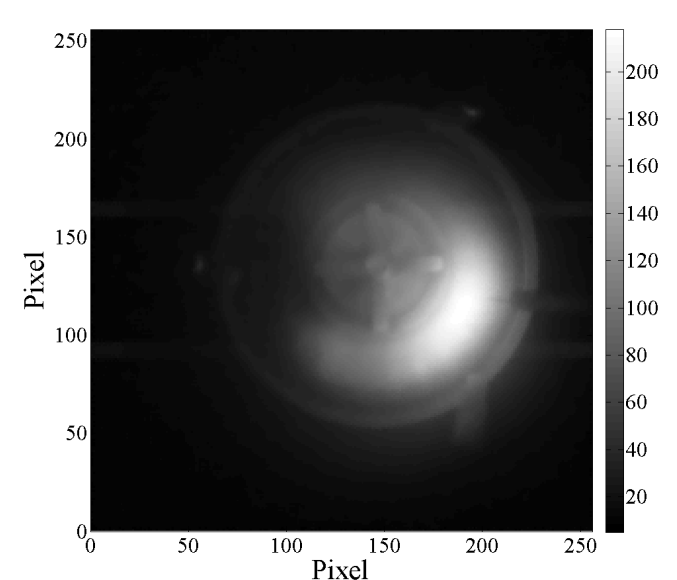

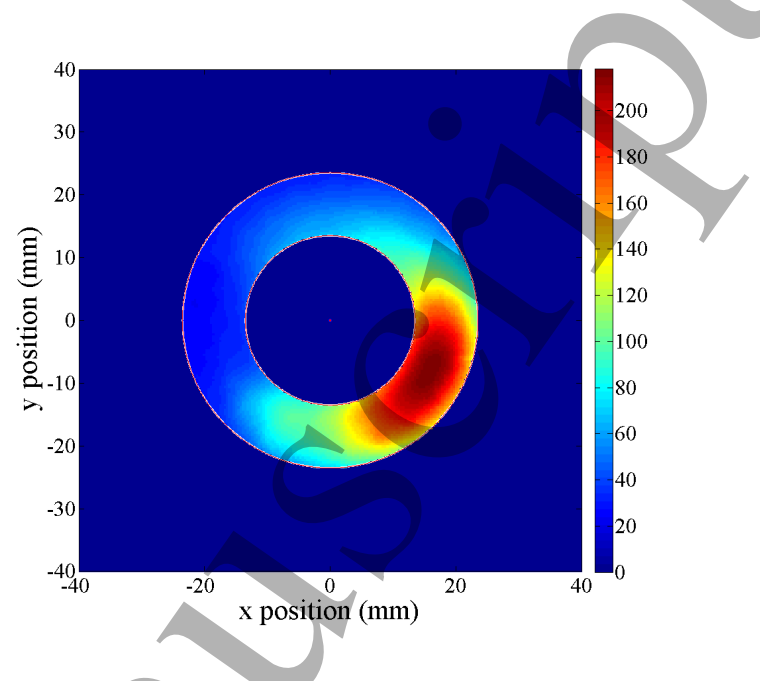

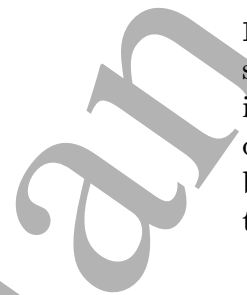

Figure 4. Color image corresponding to left raw image. Scale is in mm. A mask was applied to delete light outside the channel boundaries that are indicated by the solid lines.

format that includes all images and related information. The first step consists in transforming the cine images into TIFF (Tagged Image File Format) images, a common file format for storing raster graphics images. A TIFF file includes the images as well as tags (size, definition, image-data arrangement, applied image compression...). In this work raw cine images have been converted into 8-bit grayscale images such that each pixel is represented by one 8-bit byte. The maximum number of gray shades, or colors, is then $2^{8}=256$. The size of a TIFF video (set of 2000 images) is typically 130 MegaOctets. It is necessary to adjust the gain of the camera in such a way that the light intensity never saturates, i.e., the pixel intensity never reaches 256 .

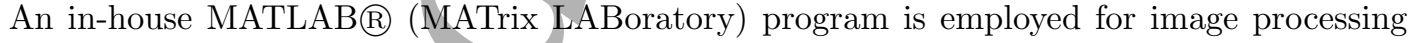
and subsequent data treatment. In order to facilitate and speed up data processing, each TIFF video must be converted into a $3 \mathrm{D}$ matrix of the form nb_pixels $\times$ nb_pixels $\times$ nb_images. A Wiener filter is used for noise reduction before copying an image into the matrix. Figure 3 shows a 8-bit gray scale filtered image of a rotating plasma inhomogeneity in the discharge of the ISCT200 Hall thruster in wall-less configuration firing at $110 \mathrm{~V}, 1.2 \mathrm{mg} / \mathrm{s}-\mathrm{Xe}$ and $0.97 \mathrm{~A}$ discharge current. The high-speed camera settings were $100000 \mathrm{fps}(10 \mu \mathrm{s}$ time interval), $7.66 \mu$ s exposure time, $256 \times 256$ pixel resolution.

The next step is the transformation of pixels into $\mathrm{mm}$ to get the real rotating structure size and speed. For this it is necessary to find the thruster center and the channel inner and outer boundaries. To simplify image processing, the light recorded outside the thruster channel boundaries is masked, equivalent to setting the light intensity to $2^{0}=1$ outside the channel. Finally, the grayscale is converted into a color scale, which aids the plasma structure visualization.

Figure 4 shows a color image obtained from the previous black and white image (Fig. 3). The $x$ and $y$ axis are in $\mathrm{mm}$ and a mask has been applied to remove light outside the channel boundaries.

4.2. Rotating structure image series

A time series of processed images of the ISCT200-WL thruster plasma discharge is shown in Fig. 5. The Hall thruster was operated at $110 \mathrm{~V}$ applied voltage, $1.3 \mathrm{mg} / \mathrm{s}$ Xe mass flow rate and $1.16 \mathrm{~A}$ discharge current. The camera frame rate was set to 100000 (time interval $=10 \mu \mathrm{s}$ ) and the exposure time was $7.66 \mu \mathrm{s}$. 
Rotating spoke instabilities in a wall-less Hall thruster: Experiments.
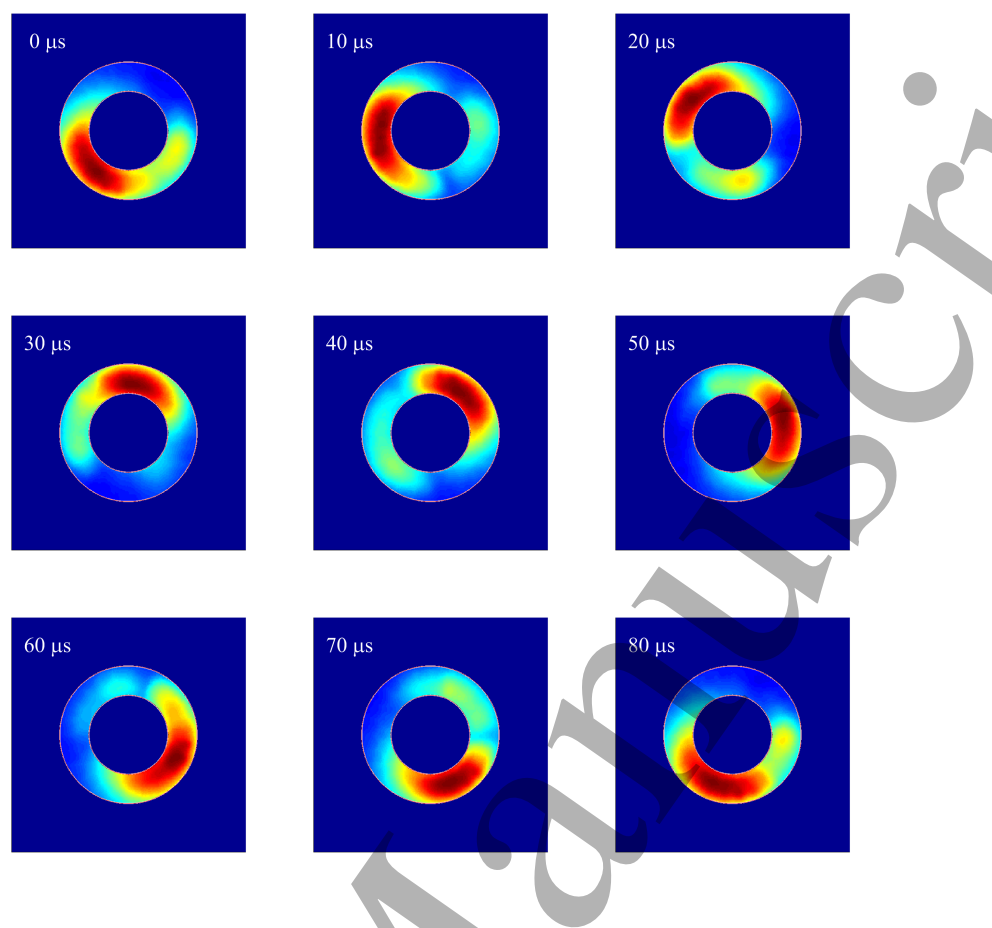

Figure 5. Time series of processed fast camera images of the ISCT200-WL thruster $(110 \mathrm{~V}, 1.2 \mathrm{mg} / \mathrm{s})$.

A well-defined structure propagating clockwise, i.e. in the $E \times B$ drift direction, is visible in Fig. 5. The bright region (in red according to our colorbar) corresponds to the plasma inhomogeneity or spoke, which is a region of high plasma density. As light is linked to excitation processes by electron impact, a luminous zone corresponds to a region with an expected high electron density and electron temperature! Only one structure is present: the discharge is said to be in the $m=1$ mode, following the definition proposed by McDonald and co-workers [15]. The mode number $m$ indicates the number of structures simultaneously present in the discharge. The $m=0$ mode corresponds to the so-called Hall thruster breathing mode [21,43-45]. In that particular case, there is a single structure that covers the entire channel cross-section area and the discharge flashes as a whole.

The structure size is about $1 / 4$ of the channel cross-section area. Its length is about $1 / 4$ of the channel circumference and its width is the channel width. However, as the camera exposure time is relatively long, the structure dimensions are likely to be somewhat overestimated.

As can be seen in Fig. 5, it takes about $90 \mu$ s for the structure to travel one complete rotation. The rotation frequency $f$ is therefore around $11.1 \mathrm{kHz}$. Notice in this work the rotation frequency is below the breathing mode frequency whatever the configuration and the operating parameters [46]. The rotation velocity is defined as:

$$
v=2 \pi r f=\pi d f
$$

where $r$ and $d$ are the channel mean radius and mean diameter, respectively. The structure velocity is then $1290 \mathrm{~m} / \mathrm{s}$, significantly lower than the electron drift velocity, or $E \times B$ speed, at the channel exit, which is $\sim 10^{6} \mathrm{~m} / \mathrm{s}$. The angular velocity $\omega$ is given by:

$$
\omega=\frac{v}{r}=2 \pi f
$$

The angular velocity is equal to $69743 \mathrm{rad} / \mathrm{s}$. The wavelength associated with the plasma inhomogeneity is:

$$
\lambda=v T=\frac{v}{f},
$$


Rotating spoke instabilities in a wall-less Hall thruster: Experiments.

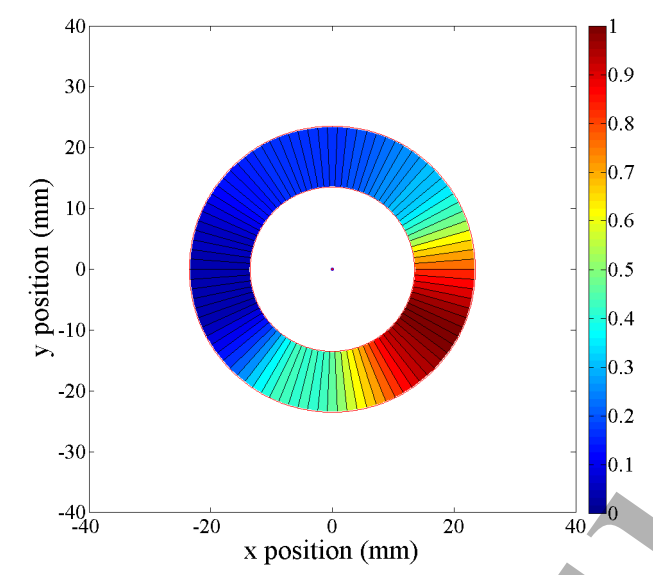

Figure 6. Normalized segmented image (90 slices) of the image shown in Fig. 4 for the ISCT200-WL thruster operating at $110 \mathrm{~V}, 1.2 \mathrm{mg} / \mathrm{s}$ and $0.97 \mathrm{~A}$ discharge current.

where $T$ is the rotation period. Here $\lambda$ is the channel circumference as $m=1$. A more general definition of the propagation velocity and wavelength accounts for the mode number:

$$
v_{m}=\frac{2 \pi r f_{m}}{m} \quad \text { and } \quad \lambda_{m}=\frac{v_{m}}{f_{m}}=\frac{2 \pi r}{m},
$$

where the subscript $m$ refers to the value of $m$.

\subsection{Video analysis}

Viewing of a series of images allows an assessment of several quantities, such as the plasma structure sizes, the rotation period and the mode number. However, to get accurate values of structure characteristics and to capture detailed information about the dynamics, the analysis of time sequences of digital images has to be/performed with mathematical tools and sophisticated

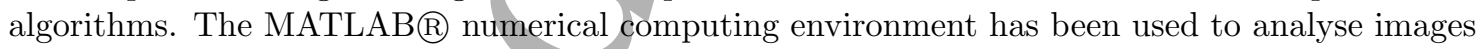
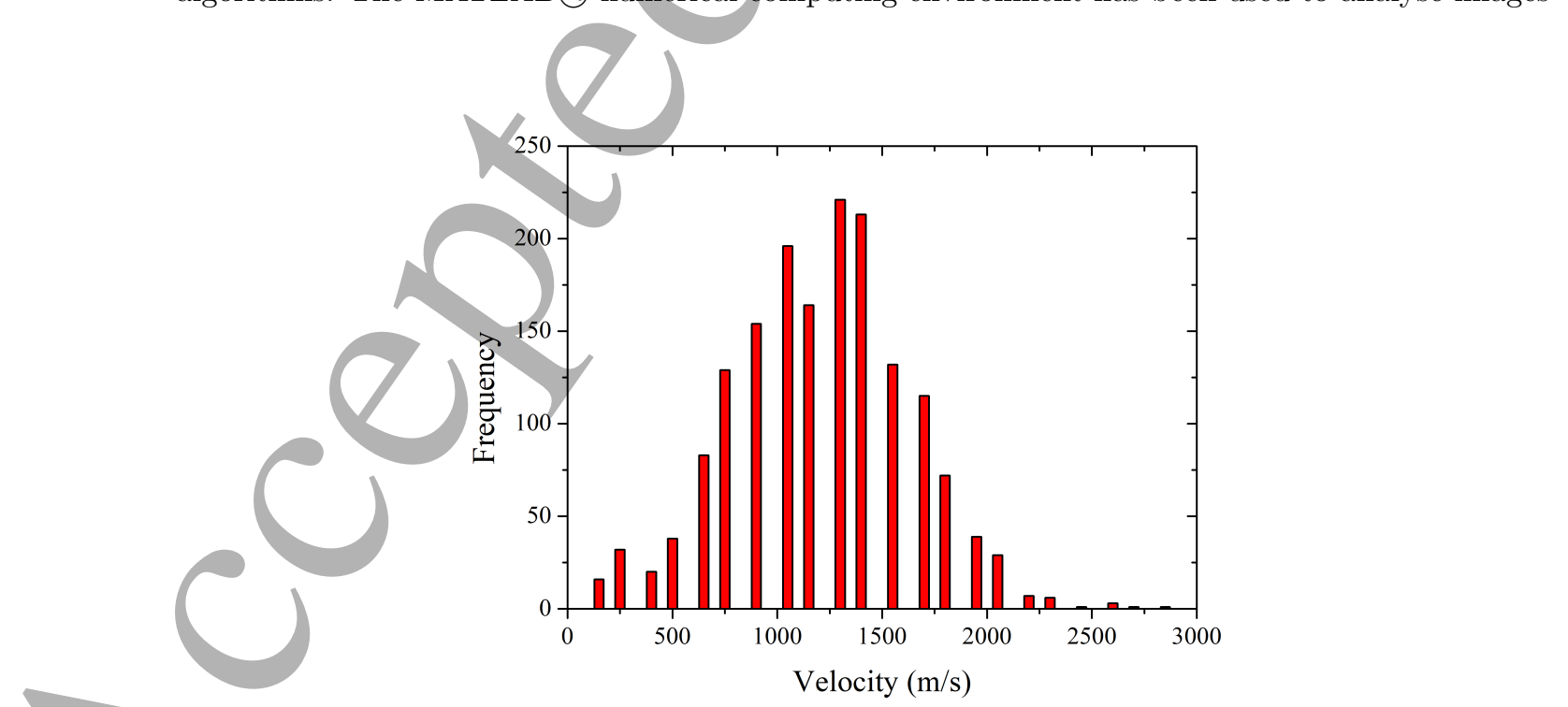

Figure 7. Velocity distribution function computed from analysis of segmented images for the ISCT200-WL thruster operating at $110 \mathrm{~V}, 1.2 \mathrm{mg} / \mathrm{s}$ and $0.97 \mathrm{~A}$ discharge current. 
and videos as it allows matrix manipulations, which is the basic form of a plasma inhomogeneity video after transformation [47]. Several methods for computing the plasma structure velocity and rotation frequency have been compared in a previous study [28]: visual inspection, temporal tracing of the light intensity, Fourier transformation of the light intensity, image segmentation and the use of spoke surfaces. All of these techniques give values in relatively good agreement, however, the segmented image method is, in this specific case, the most accurate and the easiest to implement. In the remainder of this paper the image segmentation method has therefore been used to extract plasma structure parameters from videos of the ISCT200 Hall thrusters.

To generate a segmented image, the light intensity distribution inside the area delimited by the annular channel walls is divided into discrete angular segments or slices with $n$ pixels [17]. The pixel intensity is averaged in each slice. A slice is then defined by only two quantities: angle and intensity, or color. Image segmentation is a way to reduce a 3D problem $(r, \theta$, pixel intensity) into a $2 \mathrm{D}$ problem $(\theta$, mean intensity). The number of slices can be chosen between 2 and 360 . There is, however, a minimum number below which the plasma structure is not well identified because intensity changes are too smoothed. In our case, this number is around 60 and a segmented image is typically cut into $\sim 100$ slices. A normalized segmented image of the ISCT200-WL thruster image presented in Fig. 4 is shown in Fig. 6. The channel area is divided into 90 slices.

The plasma inhomogeneity angular position is defined as the azimuthal angle of the slice with the highest intensity. Following this approach, the structure is at $-34^{\circ}$ in Fig. 6 . The rotating velocity is determined from the structure angular position of two consecutive frames:

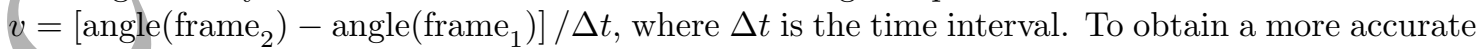
value of $v$, two constraints are included in the algorithm. Firstly, to eliminate sporadic "jumps", the difference between two angles cannot exceed a certain threshold, half a circle, i.e. $180^{\circ}$, here. Secondly, structures moving in the opposite direction, i.e. in a counterclockwise direction in the current example, are not taken into account. The treatment of each video frame therefore leads to a distribution of the rotation velocity. The plasma inhomogeneity propagation velocity distribution 
Rotating spoke instabilities in a wall-less Hall thruster: Experiments.

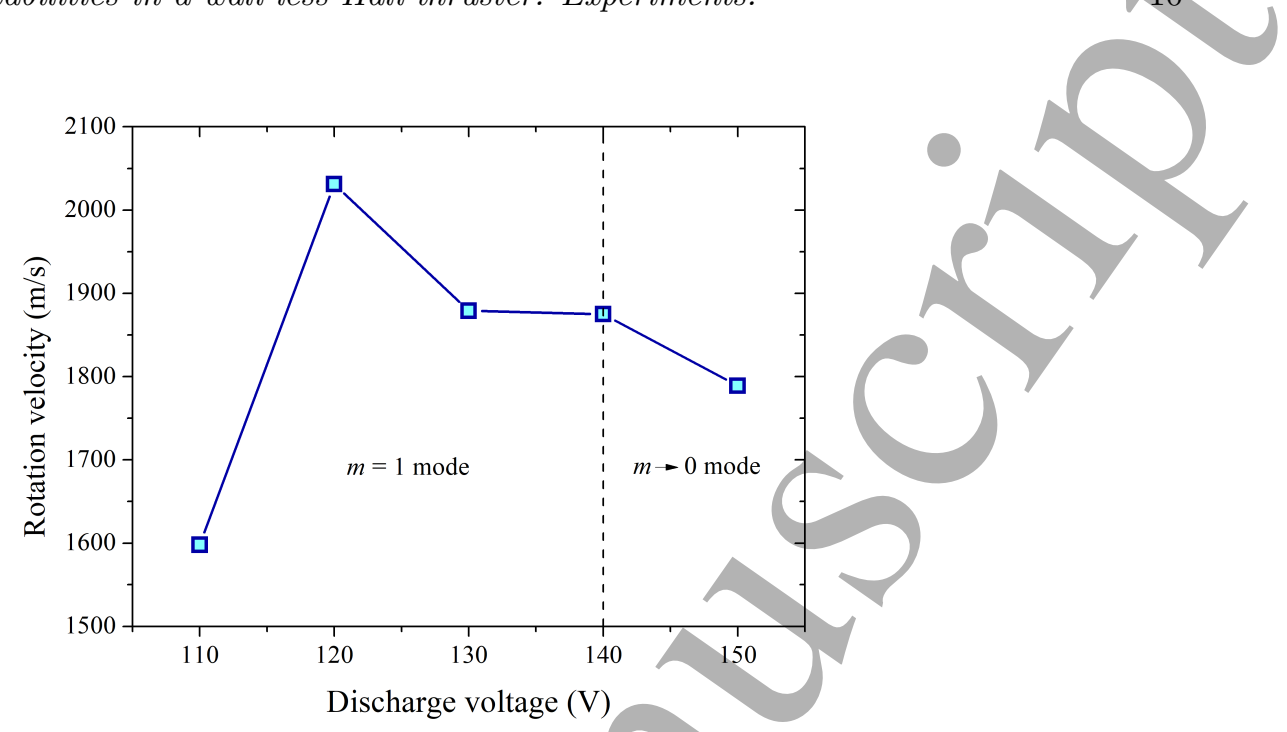

Figure 9. Plasma inhomogeneity rotation/velocity against discharge voltage for the ISCT200-ST thruster fired at $1.2 \mathrm{mg} / \mathrm{s}$.

function is shown in the form of a bar graph in Fig. 7 in the case of the ISCT200 thruster in WL configuration operating at $110 \mathrm{~V}$. The frame number was 2000 and the time interval was set to $10 \mu \mathrm{s}$. The mean velocity and the most probable velocity associated with the distribution are $1213 \mathrm{~m} / \mathrm{s}$ and $1279 \mathrm{~m} / \mathrm{s}$, respectively. As shown in [28], the slice number has little influence on the structure rotation velocity. In this work, the slice number is set to 90 .

\section{Rotating spoke properties: Standard configuration}

In recent years, several works about rotating spokes have been carried out with Hall thrusters in the standard magnetic configuration, see e.g. $[11,14-18]$. Here we summarize the main results obtained with our ISCT200 thruster in standard configuration. These results will serve as a baseline for comparisons with spoke properties in the WL configuration.

Images of the plasma discharge of the ISCT200 Hall thruster in the standard configuration have been acquired with the high-speed camera for discharge voltages ranging from $110 \mathrm{~V}$ to $400 \mathrm{~V}$ (at a xenon mass flow rate of $1.2 \mathrm{mg} / \mathrm{s}$ up to $250 \mathrm{~V}$ and a mass flow rate of $1 \mathrm{mg} / \mathrm{s}$ at higher voltages). The camera frame rate was set to $130000 \mathrm{fps}$ and the exposure time was $6.19 \mu \mathrm{s}$. Figure 8 shows a series of nine consecutive digital images of the ISCT200-ST thruster fired at $120 \mathrm{~V}$. A Wiener filter has been applied to all video frames, which have been cropped to remove pixels outside the annular channel boundaries. A well-defined rotating plasma inhomogeneity in the $m=1$ mode can be observed in this figure. The plasma structure rotates in the clockwise direction, i.e. in the $E \times B$ electron drift velocity direction. The image sequence of Fig. 8 indicates a rotation period around $55 \mu \mathrm{s}$, a frequency equal to $18180 \mathrm{~Hz}$ and a propagation velocity in the azimuthal direction that amounts to $2115 \mathrm{~m} / \mathrm{s}$, or approximately $0.2 \%$ of the $E \times B$ speed. The rotation velocity appears to be much larger than the velocity measured in the wall-less configuration at an identical voltage. The plasma structure dynamics are less stable, or less periodic, in the standard configuration of the $200 \mathrm{~W}$ Hall thruster. The dispersion in velocity is relatively large and the structure dimensions change over the course of the rotation, as illustrated in the frames shown in Fig. 8. An $m=1$ mode structure has been observed up to $U_{d} \approx 150 \mathrm{~V}$. Above this voltage the discharge is in the $m=0$ mode and only the breathing oscillation remains with a frequency around $10 \mathrm{kHz}$. The length of the plasma structure increases gradually when $U_{d}$ is ramped up, see [28]. The transition between the $m=1$ and $m=0$ modes occurs when the structure size matches the annular channel circumference. 
Rotating spoke instabilities in a wall-less Hall thruster: Experiments.
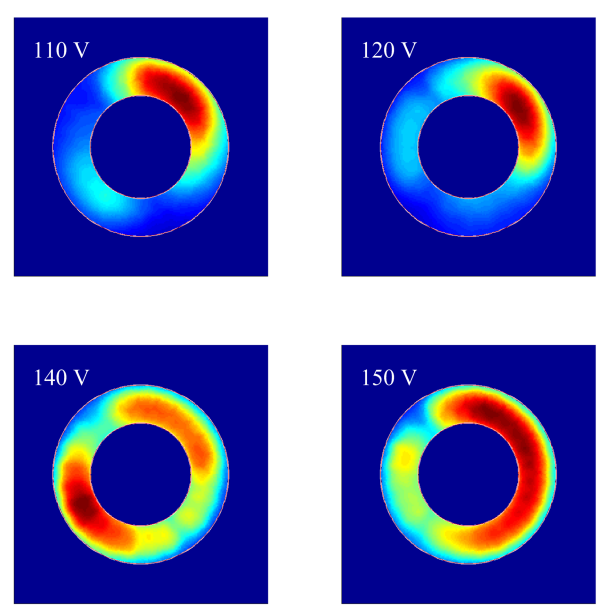

Figure 10. Digital images of th ISCT200 thruster in the wall-less configuration at various discharge voltages $(1.2 \mathrm{mg} / \mathrm{s})$.

The structure rotation velocity was determined between $110 \mathrm{~V}$ and $150 \mathrm{~V}$ using the image segmentation methods outlined previously. The xenon mass flow rate was fixed at $1.2 \mathrm{mg} / \mathrm{s}$. A plot of the plasma structure rotation velocity as a function of the voltage is shown in Fig. 9, where the velocity corresponds to the most probable velocity. The rotation velocity reaches its maximum at $U_{d}=120 \mathrm{~V}$. A possible source of uncertainty is the large size of the structure. However, other analysis techniques also reveal that the velocity increases with $U_{d}$ up to $140 \mathrm{~V}$. At this voltage, the plasma discharge enters the $m=0$ regime: the azimuthal inhomogeneity disappears and the breathing instability dominates.

\section{Rotating spoke properties: Wall-less configuration}

\subsection{Impact of the applied voltage}

Images of the plasma discharge of the ISCT200 Hall thruster in the wall-less configuration have been acquired with the high-speed camera for discharge voltages ranging from $110 \mathrm{~V}$ to $350 \mathrm{~V}$ at a xenon mass flow rate of $1.2 \mathrm{mg} / \mathrm{s}$. The camera frame rate was set to $100000 \mathrm{fps}$ and the exposure time was $7.66 \mu \mathrm{s}$. A series of consecutive digital images of the ISCT200-WL thruster fired at $110 \mathrm{~V}$ is displayed in Figure 5.

The plasma structure rotates in the clockwise direction, i.e. in the $E \times B$ electron drift velocity direction, whatever the applied voltage. The change of configuration, ST versus WL, does not affect the rotation direction, which would be expected to be governed by the magnetic field direction. The influence/ of the applied discharge voltage on the plasma inhomogeneity shape is illustrated in Fig. 10. An $m=1$ mode structure is observed up to $U_{d} \approx 200 \mathrm{~V}$, with a few occurrences of the $m=2$ mode, especially above $130 \mathrm{~V}$. However, the structure stops rotating at about $150 \mathrm{~V}$. Between $110 \mathrm{~V}$ and $130 \mathrm{~V}$, the plasma inhomogeneity is clearly-defined with sharp boundaries, and exhibits stable dynamics and quasi-periodicity with a relatively low velocity dispersion. From $150 \mathrm{~V}$ the breathing oscillation dominates with a frequency between $25 \mathrm{kHz}$ and $40 \mathrm{kHz}$ depending on the voltage [28]. A distinct $m=0$ mode with a homogeneous discharge in the azimuthal direction is observed above $200 \mathrm{~V}$. As with the standard configuration, the length of the plasma structure increases when $U_{d}$ is ramped up in the WL geometry. Fig. 10 illustrates the change in size of the structure with the applied voltage.

A plot of the plasma structure rotation velocity as a function of the voltage is displayed in Fig. 11 for the WL configuration. The velocity has been determined by means of the image segmentation method. Both the mean and the most probable velocities are shown in the figure. 
The two values are very close, indicating that the VDF is symmetrical. As with the standard configuration, there is an apparent increase in the velocity up to $U_{d}=140 \mathrm{~V}$. The rotation velocity varies from about $0.1 \%$ to $0.2 \%$ of the electron drift velocity. The velocity magnitude is nonetheless slightly lower in the WL configuration compared to the conventional configuration (see Fig. 9).

\subsection{Impact of the propellant mass flow rate}

For studying the effect of the xenon mass flow, the high-speed camera settings were $250000 \mathrm{fps}$, $128 \times 128$ image resolution and $3.286 \mu$ s exposure time. The thruster has been fired at two distinct discharge voltages, namely $110 \mathrm{~V}$ and $130 \mathrm{~V}$. The propellant mass flow rate has been varied in the range $[1.0-1.7] \mathrm{mg} / \mathrm{s}$. These limits were set by the stability of the plasma discharge.

A well-defined plasma structure in the $m=1$ mode is chiefly observed, whatever the gas flow rate for the two voltages. A few occurrences of the $m=2$ mode have been seen. The structure always propagates in the clockwise direction, i.e. in the $E \times B$ drift direction. The azimuthal velocity has been computed using the segmentation method with 90 bins. The mean velocity of the distribution is plotted in Fig. 12 as a function of the mass flow rate. The velocity increases with the mass flow rate over the entire range for $U_{d}=110 \mathrm{~V}$. When $U_{d}$ is set to $130 \mathrm{~V}$, the velocity first increases and then reaches a plateau around $2500 \mathrm{~m} / \mathrm{s}$. Changes in the rotation velocity with the gas flow rate demonstrate the influence of the neutral gas density on the plasma inhomogeneity dynamics.

\subsection{Influence of the cathode heater current}

High-speed imaging has been performed with the cathode heater current $I_{c}$ varying between $16 \mathrm{~A}$ and $19 \mathrm{~A}$. The operating conditions of the ISCT200-WL Hall thruster were $130 \mathrm{~V}$ and $1.3 \mathrm{mg} / \mathrm{s}$. When the heating power of a cathode is increased at a given gas flow rate, it is known that the discharge potential decreases if $I_{d}$ is fixed or $I_{d}$ increases when $U_{d}$ is kept constant, see [27] and references therein. Those properties are linked to the thermoionic emission phenomenon. A plot of the plasma inhomogeneity rotation velocity is displayed in Fig. 13. The heater current has a clear impact on the rotation velocity: it increases quickly when the current is ramped up. It also influences $I_{d}$ as seen in Fig. 13. The discharge current increases gradually from $1.1 \mathrm{~A}$ to $1.2 \mathrm{~A}$ when $I_{c}$ is changed from $16 \mathrm{~A}$ to $18.5 \mathrm{~A}$. It drops back to $1.1 \mathrm{~A}$ at $19 \mathrm{~A}$, which is a sign of a change 
Rotating spoke instabilities in a wall-less Hall thruster: Experiments.

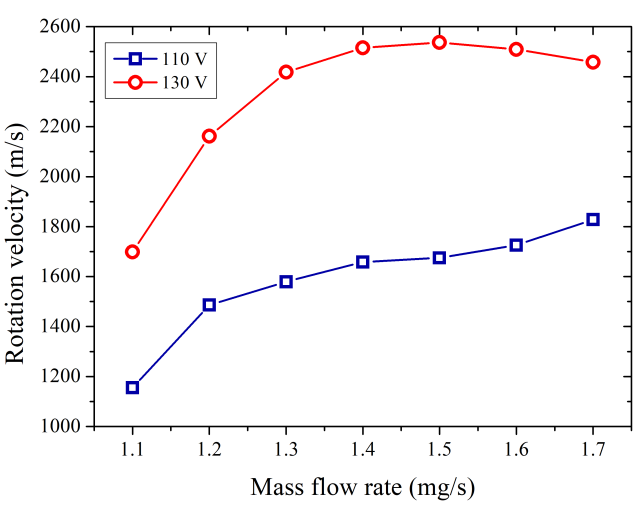

Figure 12. Plasma inhomogeneity rotation velocity versus xenon mass flow rate for the ISCT200WL thruster at $110 \mathrm{~V}$ (squares) and $130 \mathrm{~V}$ (circles).

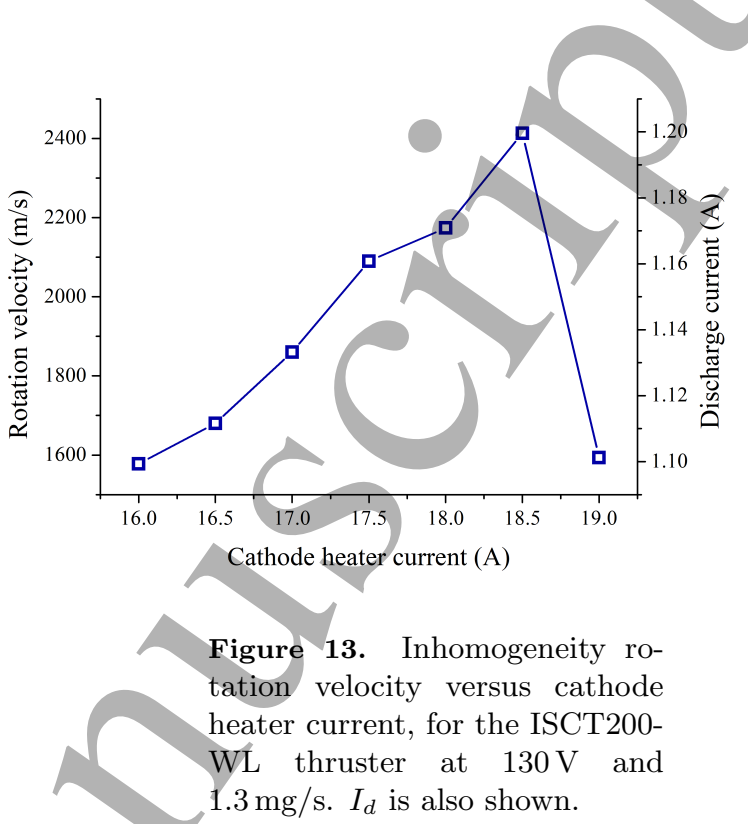

in the cathode discharge mode [27]. The sudden drop of the velocity at $19 \mathrm{~A}$ is certainly due to $I_{d}$ returning to $1.1 \mathrm{~A}$.

The plasma structure generally rotates clockwise, i,e., in the $\mathrm{E} \times \mathrm{B}$ direction, but at $16 \mathrm{~A}$ counterclockwise rotation is sometimes observed. The structure is most often in the $m=1$ mode. A few occurrences of the $m=2$ mode have been seen, but there is no apparent correlation with the cathode heater current intensity.

\section{Time evolution of the ion VDF}

\subsection{Time-resolved LIF}

In the case of time-resolved laser induced fluorescence spectroscopy (TR-LIF) the fluorescence radiation is recorded as a function of time and correlated with a periodic phenomenon occurring in the thruster discharge [41,42]. For oscillatory phenomena with characteristic frequencies in the kilohertz range, there are only on the order of ten LIF photons collected per period. To reconstruct a time-dependent ion VDF with an acceptable signal-to-noise ratio and temporal resolution, the signal therefore needs to be accumulated over tens of thousands of cycles at the very least. Different methods have been successfully used to acquire the time-varying fluorescence signal [48-51]. While TR-LIF studies on Hall thrusters have so far focused on the breathing mode instability, all approaches, however, require either a stabilization system or a fairly stable and periodic phenomenon. Unfortunately, high-speed imaging studies show that the natural plasma inhomogeneity rotation is never perfectly stable, both in frequency and phase. In the worst cases, plasma structures can appear and disappear at seemingly random times and locations. This poses a challenge for TR-LIF as whatever the method chosen, knowledge of the plasma inhomogeneity position at a specific time is required.

\subsection{Plasma inhomogeneity detection}

Attempts at stabilizing the $m=1$ plasma inhomogeneity by applying a sinusoidal frequencytunable potential on the negative pole of the cathode were unsuccessful [28]. It was therefore necessary to find a way to directly detect the inhomogeneity angular position. One obvious way is to simultaneously acquire the LIF signal and high-speed video. However, this quickly turns out to be impractical due to the size and transfer time of the video files. Instead of observations of the whole thruster channel cross-section area, the light intensity was captured at the LIF measurement 
Rotating spoke instabilities in a wall-less Hall thruster: Experiments.
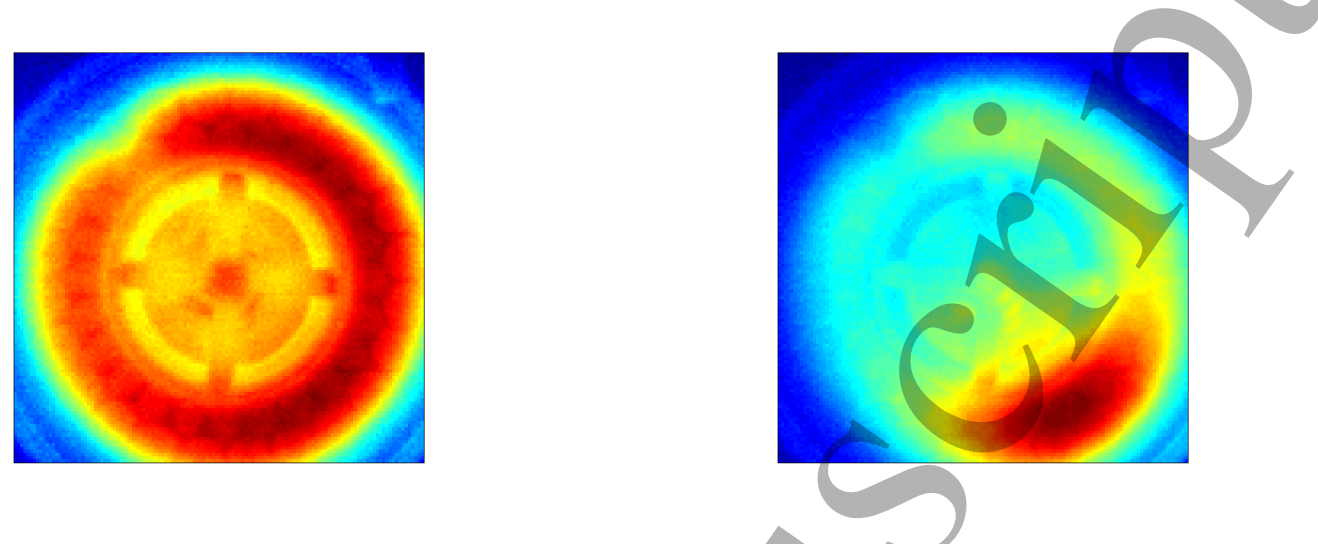

Figure 14. Image obtained from 129 frames without synchronization to the LIF setup PMT signal (left). Image constructed from the same 129 frames with synchronization. ISCT200-WL thruster fired at $110 \mathrm{~V}$.

location (channel axis, $x=4 \mathrm{~mm}$ and $\theta=-90^{\circ}$ ). The collection branch optical fiber was split in two. A second photomultiplier tube was used to record the total light intensity in the measurement volume. A $10 \mathrm{~nm}$ bandwidth interference filter was placed between the fiber output and the second detector to isolate the ion fluorescence line at/542 nm.

When a $m=1$ rotating spoke is present, the light trace shows a periodic oscillation with a frequency identical to the plasma inhomogeneity rotation frequency. The light signal, i.e. photomultiplier output, peak can be isolated using a simple peak detect algorithm and subsequently used to pick the corresponding frames in the synchronized high speed video. Figure 14 (right) shows an image built with 129 of such frames. The plasma structure is well localized, which demonstrates that the observed peaks in the light waveform correspond to the passage of a plasma inhomogeneity. This method, i.e. the use of the photomultiplier tube along with the LIF signal, allows us to simultaneously determine when the plasma inhomogeneity crosses the LIF measurement volume and when a given photon from ion induced fluorescence is generated.

\subsection{Data acquisition}

Data acquisition based on the aforementioned method requires special attention. In order to capture single photons the setup has to be able to detect pulses with $\mathrm{a} \approx 10 \mathrm{~ns}$ width. The fluorescence signal must be recorded over tens of thousands of cycles, i.e., during several seconds, to get an accurate assessment of the shape of the fluorescence signal over one cycle. In order to get both the sample rate and memory depth required, a Lecroy HD6104 oscilloscope was used instead of our MCS-PCI pulse counter [48]. The characteristics of the oscilloscope are 12-bit resolution, $1 \mathrm{GHz}$ bandwidth, $250 \mathrm{Mpts}$ per channel and a $1.25 \mathrm{GS} / \mathrm{s}$ sampling rate. The oscilloscope records single photons from the two photomultiplier tubes, i.e. LIF light and total fluorescence light, as well as the signal from a fast photodiode that indicates if the laser beam propagates through the plasma (mode $\mathrm{ON})$ or is suspended (mode OFF). The cw laser light modulation is achieved with a mechanical chopper set at about $1 \mathrm{kHz}$. When the laser is ON, respectively OFF, the signal includes LIF and background photons, respectively, background photons only. The LIF signal is then obtained by substracting the ON and OFF signals [41]. With a sampling frequency of $10 \mathrm{MHz}$ each individual record lasts 1 second. Accumulation of about $100 \mathrm{~s}$ of recording is necessary to obtain suitable ion VDFs with a good signal-to-noise ratio. Examples of raw data recorded by the oscilloscope are shown in Fig. 15. The procedure to obtain the time-dependent ion VDF at a given position consists in accumulating photons in time at a fixed wavelength, i.e. in recording the time evolution of a well-defined ion velocity group.

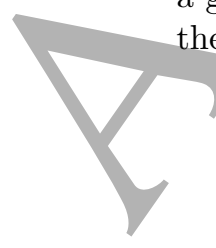




\subsection{Data treatment and VDF construction}

The first step in the data processing is the detection of each photon. For this, a peak detection algorithm is used and only peaks of a certain minimum amplitude are retained. This discrimination step allows the elimination of detector dark noise photons. The photodiode signal is then read to determine if those photons were collected during the ON or the OFF phase of the laser cycle. From this information alone, it is possible to reconstruct the averaged shape of the ion VDF. For each wavelength, the number of photons collected per second when the laser is OFF is subtracted from that when the laser is ON. The photon number per second is used, rather than the total number of photons, because the durations of the ON and OFF periods are not exactly equal. The validity of this photon-counting technique is established by comparing the time-averaged ion
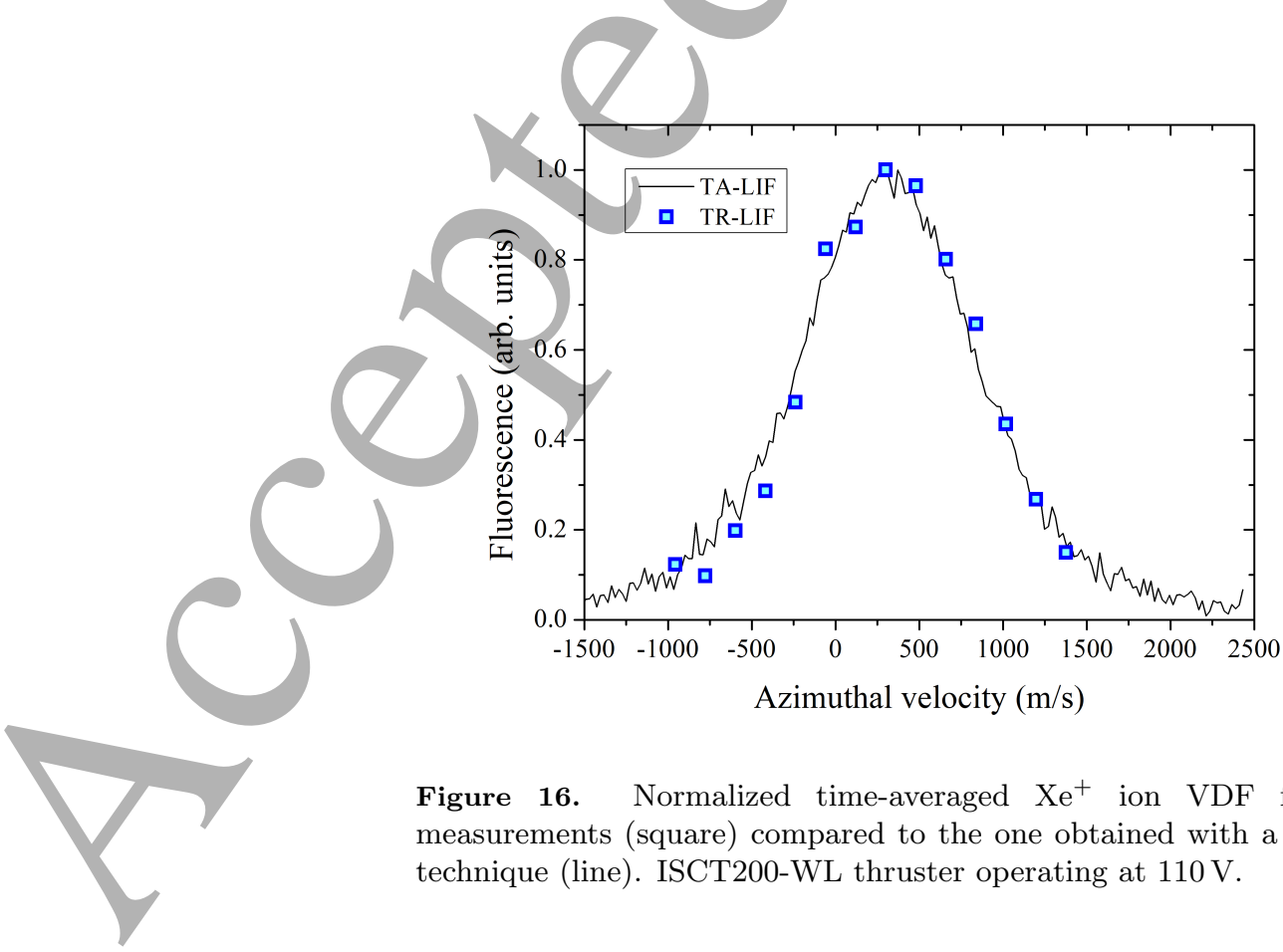

Figure 16. Normalized time-averaged $\mathrm{Xe}^{+}$ion VDF from time-resolved LIF measurements (square) compared to the one obtained with a standard phase-sensitive technique (line). ISCT200-WL thruster operating at $110 \mathrm{~V}$. 
VDF obtained by means of a lock-in detector with that built from the temporal average of timevarying fluorescence traces. The result is illustrated in Fig. 16. As is apparent from this figure, the temporally-averaged ion VDF does not depend on the fluorescence radiation acquisition method. The two ion VDFs have a very similar shape and the most probable azimuthal velocity is the same: $v_{\mathrm{mp}}=300 \mathrm{~m} / \mathrm{s}$. This result indicates that the current statistics for the acquisition duration are adequate. Wings of the fluorescence profile are not captured in this example, meaning that the number of probed velocity groups has to be increased.

Once each photon has been identified for a particular instant in time and a corresponding laser cycle (ON versus $\mathrm{OFF})$, their position in a plasma inhomogeneity rotation cycle may be determined. The temporal range of each plasma inhomogeneity passage is found using the method previously described. From that, the delay between the plasma inhomogeneity and photons before and after the structure has crossed the measurement volume is computed, with this precise moment taken as a reference. This operation provides the number of photons as a function of time for the $\mathrm{ON}$ and OFF modes. Subtracting the two signals for each laser wavelength gives the time-varying ion VDF.

\subsection{Time-varying $\mathrm{Xe}^{+}$ion $\mathrm{VDF}$}

The time-varying ion VDF has been measured in the discharge of the ISCT200-WL thruster with a long acquisition period in order to provide a large signal-to-noise ratio. The Hall thruster operating conditions were $110 \mathrm{~V}, 1.3 \mathrm{mg} / \mathrm{s}$ and $1.15 \mathrm{~A}$ discharge current. Time-resolved LIF measurements of the azimuthal velocity component have been performed on the channel centerline $4 \mathrm{~mm}$ downstream of the anode at $-90^{\circ}$, i.e. in the ionization region (see $[36,40]$ ). The timedependent $\mathrm{Xe}^{+}$ion VDF is shown in Fig. 17 along with the relative ion density that correspond to the LIF signal intensity corrected for the laser power variation. The $t=0$ reference time refers to the detection of the plasma structure. Under these thruster operating conditions, the $m=1$ inhomogeneity rotation period is about $70 \mu \mathrm{s}$ (see Section 6). The ion VDF does not change much over time, with an amplitude that scales with density. Most ions rotate in the $\mathrm{E} \times \mathrm{B}$ direction (positive velocity), i.e. in the direction of the spoke motion. However, some ions propagate in the opposite direction (negative velocity) especially when the ion density is large, as can be seen in 
Fig. 17. The mean ion velocity does not vary significantly. The highest magnitude of the mean ion velocity is around $+400 \mathrm{~m} / \mathrm{s}$. It corresponds to an ion thermal speed; in that case one finds $T_{i}=1000 \mathrm{~K}$, a reasonable value for a low-power Hall thruster [41]. The measured ion velocity is well below the rotating spoke velocity, which is about $1600 \mathrm{~m} / \mathrm{s}$, and the ion sound speed that reaches $3820 \mathrm{~m} / \mathrm{s}$ with $T_{e}=20 \mathrm{eV}$, in agreement with previous work by Sekerak in high-power Hall thrusters [46].

\subsection{Simulated ion VDF}

Computer simulations of the plasma discharge of the ISCT200 Hall thruster in the wall-less configuration have been performed using the self-consistent 3D-3V Particle-In-Cell code STOIC (electroSTatic Optimized particle In cell). A detailed description of the STOIC code can be found in references [52-54]. Only the main parameters are given here. Simulations are performed on a $70 \times 70 \times 50$ grid. The total number of computational particles is around $2 \times 10^{7}$. The cell size is $0.1 \mathrm{~mm}$ in all directions, smaller than the Debye length everywhere. The time step is set to $5.6 \times 10^{-12} \mathrm{~s}$ to resolve the plasma and cyclotron frequencies. Scaling laws are used to reduce the computational time. Information and results about numerical simulations of a wall-less Hall thruster are available in $[55,56]$. For this work, a specific diagnostic module has been developed and implemented in the code to enable the monitoring of the atom and ion VDF in time and space.

Figure 18 shows the simulated temporal evolution of ion density, ion velocity distribution function in the azimuthal direction and local plasma potential during two rotation cycles of the plasma structure at $x=4 \mathrm{~mm}, y=0 \mathrm{~mm}$ (on-axis) and $\theta=-90^{\circ}$. The simulation conditions were $1 \mathrm{mg} / \mathrm{s}$ Xe flow rate, $175 \mathrm{~V}$ discharge voltage and a neutral gas temperature of $400 \mathrm{~K}$. In Fig. 18 the velocity is expressed as a multiple of the local ion acoustic wave speed $c_{s}$, which is equal to $3834 \mathrm{~m} / \mathrm{s}$ here. As can be seen in Fig. 18, the distribution of the ion azimuthal velocity component is most often distinctly non-Maxwellian, with high-velocity tails. High-energy ions are created 
more specifically at the edges of the rotating spoke, where strong potential gradients are present.

The measured and simulated time-dependent $\mathrm{Xe}^{+}$ion VDF are in relatively good agreement, although the thruster operating conditions are different, particularly the applied voltage [55]. The code reproduces the rotating spoke properties in terms of size, mode number, drift direction and dynamics, though the $m=1$ to $m=0$ mode transition occurs at higher voltages around $250 \mathrm{~V}$. Similarly, the simulated plasma inhomogeneity rotation velocity in the azimuthal direction is larger than that inferred from fast camera imaging. The discrepancy may originate from scaling relations used in the PIC simulations to reduce the computational time and/or from the applied boundary conditions.

Many characteristics of the simulated ion VDF also appear on the measured distribution function. The mean $\mathrm{Xe}^{+}$ion azimuthal velocity is positive, i.e. in the $E \times B$ direction, except at the plasma structure boundaries, with a small amplitude, well below the structure rotation velocity. Simulations give a plasma structure rotation velocity of $2.9 \mathrm{~km} / \mathrm{s}(27 \mathrm{kHz}$ rotation frequency) and a mean ion azimuthal velocity around $800 \mathrm{~m} / \mathrm{s}$ in the high density region for conditions of Fig. 18. Fast ions, with positive and negative velocities, are created during short time periods due to the the strong potential gradients at the structure boundaries. It is worth noting that very fast ions that exist at the edges of the rotating spoke are not experimentally observed by LIF spectroscopy as the associated density is low (see Fig. 18). Finally, the ion density distribution along the channel mean circumference is well-reproduced.

\section{Conclusion}

Characteristics and dynamics of rotating spokes have been investigated in the plasma discharge of a $200 \mathrm{~W}$-class permanent magnet Hall thruster in the wall-less configuration with a curved gridded anode at the channel exit plane. Such a configuration shifts the plasma discharge outside the dielectric channel. These results have been compared to those for a Hall thruster operated in the standard configuration, where the anode is placed at the back of the channel. The magnetic field topology was the same for the two configurations, with the highest magnitude at the channel outlet.

High-speed camera imaging combined with sophisticated image processing allowed the observation of the plasma structure dynamics and the determination of the mode number, the rotation frequency and the propagation velocity. For the two configurations, the $m=1$ mode dominates at low voltages and transition from this mode to the $m=0$ mode, which corresponds to the breathing oscillation, occurs around $150 \mathrm{~V}$. The plasma instabilities always rotate in the $E \times B$ electron drift velocity direction. We note that spoke rotation in the $-E \times B$ direction has indeed been observed recently in other devices [57]; it is interesting to consider whether any particular operating conditions may lead to a similar observation in Hall thrusters. To our knowledge, no such observation has yet been made.

The azimuthal spoke velocity is influenced by the applied voltage, the propellant mass flow rate and the external cathode properties. Whatever the thruster operating conditions, the rotation velocity of the plasma inhomogeneity remains below the critical ionization velocity, which is equal to $4220 \mathrm{~m} / \mathrm{s}$ for xenon. The time-varying $\mathrm{Xe}^{+}$ion velocity distribution function has been measured on the channel axis at $-90^{\circ}$ in front of the anode of the ISCT200-WL thruster at $110 \mathrm{~V}$ using a photon counting LIF spectroscopy technique. Most ions rotate in the in the $E \times B$ direction. The mean ion velocity corresponds to the thermal speed. It is well below the plasma structure rotation velocity and the ion sound speed. The ion VDF reveals the presence of ions with positive and negative velocities inside the spoke.

Numerical simulations of the rotating structure in the ISCT200-WL discharge have been performed with a 3D-3V PIC code. Several qualitative similarities have been found between experiments and simulations: identical structure sizes (about $1 / 5$ of the channel mean circumference), dominance of the $m=1$ mode whatever the operating parameters, and the transition from $m=1$ to $m=0$ at high voltages. Rotation frequencies and propagation velocity are, however, different in simulations and experiments; simulations give larger values. 
Rotating spoke instabilities in a wall-less Hall thruster: Experiments.

Discrepancies could originate from boundary conditions or scaling factors used in the $3 \mathrm{D}-3 \mathrm{~V}$ simulations. In addition, the time-dependent ion VDF in the azimuthal direction has been reconstructed from the numerical data set. There is a good qualitative agreement befween the simulated and the measured ion VDF. Simulations, however, reveal the existence of high-energy tails at the structure edges, regions of low plasma density and steep plasma potential gradients, features which will be of interest for future experimental study.

\section{Acknowledgments}

This research was funded by the EOARD under Grant FA9550-15-1-0281. We are indebted to Dr. Nabiha Chaumeix and her team for lending us the high-speed cameras.

\section{References}

[1] J. P. Boeuf. Rotating structures in low temperature magnetized plasmas - insight from particle simulations. Frontiers in Physics, 2:74:1-17, 2014.

[2] J.-P. Boeuf and B. Chaudhury. Rotating instability in low-temperature magnetized plasmas. Phys. Rev. Lett., 111:155005, 2013.

[3] P. A. Redhead. Instabilities in crossed-field discharges at low pressures. Vacuum, 38:901-906, 1998.

[4] M. Keidar and I. I. Beilis. Electron transport phenomena in plasma devices with $\mathrm{E} \times \mathrm{B}$ drift. IEEE Trans. Plasma Sci., 34:804-814, 2006.

[5] A. R. Pal, J. Chutia, and H. Bailung. Observation of instability in presence of $\mathrm{E} \times \mathrm{B}$ flow in a direct current cylindrical magnetron discharge plasma. Phys. Plasmas, 11:4719-4726, 2004.

[6] N. Brenning, D. Lundin, T. Minea, C. Costin, and C. Vitelaru. Spokes and charged particle transport in HiPIMS magnetrons. J. Phys. D: Appl. Phys., 46:084005, 2013.

[7] J. Winter, A. Hecimovic, T. de los Arcos, M. Böke, and V. Schulz von der Gathen. Instabilities in high-power impulse magnetron plasmas: from stochasticity to periodicity. J. Phys. D: Appl. Phys., 46:084007, 2013.

[8] A. Anders, P. Ni, and A. Rauch. Drifting localization of ionization runaway: unraveling the nature of anomalous transport in high power impulse-magnetron sputtering. J. Appl. Phys., 111:053304, 2012.

[9] A. Hecimovic. Anomalous cross-B field transport and spokes in HiPIMS plasma. J. Phys. D: Appl. Phys., 49:18LT01, 2016.

[10] S. Tsikata and T. Minea. Modulated electron cyclotron drift instability in a high-power pulsed magnetron discharge. Phys. Rev. Lett., 114:185001, 2015.

[11] M. E. Griswold, C. L. Ellison, Y. Raitses, and N. J. Fisch. Feedback control of an azimuthal oscillation in the E $\times$ B discharge of Hall thrusters. Phys. Plasmas, 19:053506, 2012.

[12] C. L. Ellison, Y. Raitses, and N. J. Fisch. Fast camera imaging of hall thruster ignition. IEEE Trans. Plasma Sci., 39:2950-2951, 2011.

[13] C. L. Ellison, Y. Raitses, and N. J. Fisch. Cross-field electron transport induced by a rotating spoke in a cylindrical Hall thruster. Phys. Plasmas, 19:013503, 2012.

[14] B. A. Jorns and R. R. Hofer. Plasma oscillations in a 6-kW magnetically shielded Hall thruster. Phys. Plasmas, 21:053512, 2014 .

[15] M. McDonald, C. K. Bellant, B. A. St. Pierre, and A. D. Gallimore. Measurement of cross-field electron current in a Hall thruster due to rotating spoke instabilities. In Proceedings of the 47th Joint Propulsion Conference, AIAA paper 2011-5810, San Diego, CA, 2011.

[16] M. McDonald and A. D. Gallimore. Rotating spoke instabilities in Hall thrusters. IEEE Trans. Plasma Sci., 39:2952-2953, 2011.

[17] M. S. McDonald and A. D. Gallimore. Comparison of breathing and spoke mode strength in the H6 Hall thruster using high speed imaging. In Proceedings of the 33rd International Electric Propulsion Conference, IEPC paper 2013-353, Wáshington D.C., 2013.

[18] M. J. Sekerak, A. D. Gallimore, D. L. Brown, R. R. Hofer, and J. E. Polk. Mode transitions in Hall effect thrusters induced by variable magnetic field strength. J. Propul. Power, 33:903-917, 2016.

[19] S. Jaeger, T. Pierre, and C. Rebont. Direct observation of a cross-field current-carrying plasma rotating around an unstable magnetized plasma column. Phys. Plasmas, 16:022304, 2009.

[20] C. Rebont, N. Claire, T. Pierre, and F. Doveil. Ion velocity distribution function investigated inside an unstable magnetized plasma exhibiting a rotating nonlinear structure. Phys. Rev. Lett., 106:225006, 2011.

[21] V. V. Zhurin, H. R. Kaufmann, and R. S. Robinson. Physics of closed drift thrusters. Plasma Sources Sci. Technol., 8:R1-R20, 1999.

[22] J.-P. Boeuf. Tutorial: Physics and modeling of Hall thrusters. J. Appl. Phys., 121:011101, 2017.

[23] S. Mazouffre. Electric propulsion for satellites and spacecraft: Established technologies and novel approaches. Plasma Sources Sci. Technol., 25:033002, 2016.

[24] S. Mazouffre, G. Bourgeois, K. Dannenmayer, and A. Lejeune. Ionization and acceleration processes in a small, variable channel width, permanent-magnet Hall thruster. J. Phys. D: Appl. Phys., 45:185203, 2012.

[25] A. Lejeune, G. Bourgeois, and S. Mazouffre. Kr II and Xe II axial velocity distribution functions in a cross-field ion source. Phys. Plasmas, 19:073501, 2012. 
Rotating spoke instabilities in a wall-less Hall thruster: Experiments.

[26] L. Grimaud and S. Mazouffre. Performance comparison between standard and magnetically shielded 200 W Hall thrusters with BN-SiO2 and graphite channel walls. Vacuum, 155:514-523, 2018.

[27] R. Joussot, L. Grimaud, and S. Mazouffre. Examination of a 5 A-class cathode with a LaB6 flat disk emitter in the 2A-20A current range. Vacuum, 146:52-62, 2017.

[28] S. Mazouffre, L. Grimaud, S. Tsikata, K. Matyash, and R. Schneider. Investigation of rotating spoke instabilities in a wall-less Hall thruster. Part I: Experiments. In Proceedings of the 35th International Electric Propulsion Conference, IEPC paper 2017-248, Atlanta, Georgia, 2017.

[30] L. Grimaud and S. Mazouffre. Ion behavior in low-power magnetically shielded and unshielded Hall thrusters. Plasma Sources Sci. Technol., 26:055020, 2017.

[31] I. G. Mikellides, I. Katz, R. R. Hofer, D. M. Goebel, and K. DeGrys. Magnetic shielding of the channel walls in a Hall plasma accelerator. Phys. Plasmas, 18:033501, 2011.

[32] I. G. Mikellides, I. Katz, R. R. Hofer, and D. M. Goebel. Magnetic shielding of walls from the unmagnetized ion beam in a Hall thruster. Appl. Phys. Lett., 102:023509, 2013.

[33] I. G. Mikellides, I. Katz, R. R. Hofer, and D. M. Goebel. Magnetic shielding of Hall thrusters at high discharge voltages. J. Appl. Phys., 116:053302, 2014.

[34] R. W. Conversano, D. M. Goebel, R. R. Hofer, I G. Mikellides, and R. E. Wirz. Performance analysis of a low-power magnetically shielded hall thruster: Experiments. J. Propul. Power, 33:975-983, 2017.

[35] R. W. Conversano, D. M. Goebel, I. G. Mikellides, R. R. Hofer, and R. E. Wirz. Performance analysis of a low-power magnetically shielded hall thruster: Computational modeling. J. Propul. Power, 33:992-1001, 2017.

[36] S. Mazouffre, S. Tsikata, and J. Vaudolon. Development and experimental characterization of a wall-less Hall thruster. J. Appl. Phys., 116:243302, 2014.

[37] J. Vaudolon, S. Mazouffre, C. Hénaux, D. Harribey, and A. Rossi. Optimization of a wall-less Hall thruster. Appl. Phys. Letter, 107:174103, 2015.

[38] B. Karadag, S. Cho, and I. Funaki. Thrust performance, propellant ionization, and thruster erosion of an external discharge plasma thruster. J. Appl. Phys., 123:153302, 2018.

[39] B. Karadag, S. Cho, I. Funaki, Y. Hamada, and K. Komurasaki. External discharge plasma thruster. J. Propul. Power, 34:1094-1096, 2018.

[40] S. Mazouffre, S. Tsikata, and J. Vaudolon. Development and characterization of a wall-less Hall thruster. In Proceedings of the 50th Joint Propulsion Conference, AIAA paper 2014-3513, Cleveland, OH, 2014.

[41] S. Mazouffre. Laser-induced fluorescence diagnostics of the cross-field discharge of Hall thrusters. Plasma Sources Science and Technology, 22:013001, 2013.

[42] S. Mazouffre. Laser-induced fluorescence spectroscopy applied to electric thrusters. STO-AVT-VKI Lecture series 263, Electric propulsion systems. Von Karman Institute, Edited by T. Magin, 2016. pp. 10-1..26.

[43] E. Y. Choueiri. Plasma oscillations in Hall thrusters. Phys. Plasmas, 8:1411-1426, 2001.

[44] J. P. Boeuf and L. Garrigues. Low frequency oscillations in a stationary plasma thruster. J. Appl. Phys., 84:3541-3554, 1998.

[45] S. Barral and E. Ahedo. Low-frequency model of breathing oscillations in Hall discharges. Phys. Rev. E, 79:046401, 2009.

[46] M.J. Sekerak, B. W. Longmier, A. D. Gallimore, D. L. Brown, R. R. Hofer, and J. E. Polk. Azimuthal spoke propagation in hall effect thrusters. IEEE Trans. Plasma Sci., 43:72-85, 2015.

[47] A. McAndrew. An Introduction to Digital Image Processing With Matlab. Course Technology Press, Boston, MA, 2004.

[48] J. Vaudolon, L. Balika, and S. Mazouffre. Photon counting technique applied to time-resolved laser-induced fluorescence measurements on a stabilized discharge. Rev. Sci. Instrum., 84:073512, 2013.

[49] J. Vaudolon and S. Mazouffre. Observation of high-frequency ion instabilities in a cross-field plasma. Plasma Sources Sci. Technol., 24:032003, 2015.

[50] A. L. Fabris, C. Young, and M. A. Cappelli. Time-resolved laser-induced fluorescence measurement of ion and neutral dynamics in a Hall thruster during ionization oscillations. J. Appl. Phys., 118:233301, 2015.

[51] A. Diallo, S. Keller, S. Shi, Y. Raitses, and S. Mazouffre. Time-resolved ion velocity distribution in a cylindrical Hall thruster: Heterodyne-based experiment and modeling. Rev. SCI. Instrum., 86:033506, 2015.

[52] D. Tskhakaya, K. Matyash, R. Schneider, and F. Taccogna. The Particle-In-Cell Method. Contrib. Plasma Phys., 47:563-594, 2007.

[53] K. Matyash, R. Schneider, O. Kalentev, Y. Raitses, and N. J. Fisch. Numerical investigations of a cylindrical Hall thruster. In Proceedings of the 32nd International Electric Propulsion Conference, IEPC paper 2011070, Wiesbaden, Germany, 2011.

[54] K. Matyash, R. Schneider, S. Mazouffre, Y. Raitses, and A. Diallo. 3D simulation of rotating spoke in a Hall thruster. In Proceedings of the 33rd International Electric Propulsion Conference, IEPC paper 2013-307, Washington, D.C., 2013.

[55] K. Matyash, R. Schneider, S. Mazouffre, L. Grimaud, and S. Tsikata. Investigation of rotating spoke instabilities in a wall-less Hall thruster. Part II: Simulation. In Proceedings of the 35rd International Electric Propulsion Conference, IEPC paper 2017-403, Atlanta, Georgia, 2017.

[56] K. Matyash, R. Schneider, S. Mazouffre, L. Grimaud, and S. Tsikata. Rotating spoke instabilities in a wall-less Hall thruster: Simulations. Plasma Sources Sci. Technol., Submitted, 2018.

[57] Y. Yang, J. Liu, L. Liu, and A. Anders. Propagation direction reversal of ionization zones in the transition 
1

2

3

4

5

6

7

8

9

10

11

12

13

14

15

16

17

18

19

20

21

22

23

24

25

26

27

28

29

30

31

32

33

34

35

36

37

38

39

40

41

42

43

44

45

46

47

48

49

50

51

52

53

54

55

56

57

58

59

60

Rotating spoke instabilities in a wall-less Hall thruster: Experiments.

between high and low current magnetron sputtering. Appl. Phys. Lett., 105:254101, 2014. 